\title{
EFFECT OF DC BIASED VOLTAGE AND ION TEMPERATURE ON BOUNDED ION-ION PLASMA
}

\author{
Anish Maskey, Atit Deuja, Suresh Basnet ${ }^{*}$, Raju Khanal \\ Central Department of Physics, Tribhuvan University, Kirtipur, Kathmandu 44613, Nepal \\ "Corresponding author: sbplasma1986@gmail.com,sbasnet@tucdp.edu.np
}

(Received: February 12, 2020; Revised: April 23, 2020; Accepted: April 30, 2020)

\begin{abstract}
A one dimensional particle-in-cell (PIC) simulation method has been employed to study the effect of DC voltage and ion temperature on the properties of ion-ion plasma bounded by two symmetrical but oppositely biased electrodes. It is assumed that the ion-ion plasma is collisionless and both the positive and negative ion species have the same mass, temperature, and degree of ionization. Simulation results show that the formation of sheath and presheath regions and fluctuation of plasma parameters in that region are affected by the biasing voltage and ion temperature. It was found that the magnitude of the electrostatic electric field at the vicinity of biasing electrodes was affected by the biasing voltage and ion temperature as well. This strong electric field close to the electrodes further prevents the flow of charged particles towards the electrodes. The presence of a non-zero electric field at the quasineutral region suggests a presheath region similar to the electron-ion plasma. In the quasineutral region, the density of ions increased with the increase in biasing voltage and decreased with the increase in temperature of isothermal ions. Furthermore, the phase space diagrams for the ions were obtained which indicated different regions of the plasma. The positive ions acquire negative velocity towards the negatively biased electrode and the negative ions acquire positive velocity towards the positively biased electrode.
\end{abstract}

Keywords: Biased electrodes, electronegative plasma, PIC simulation, plasma sheath, presheath

\section{INTRODUCTION}

Electronegative plasmas, which consist of negative ions, have a wide range of applications such as in electronic industry, thin film deposition, etching, etc. (Franklin, 2002). For example; plasma with negative ions $\mathrm{Cl}_{2}$ or $\mathrm{SF}_{6}$ are used for etching, oxygen plasma is commonly used to clean the non-metal materials (Economou, 2014). Besides these, the electronegative plasma is also useful to study the ionosphere plasma phenomenon (Chapagain, 2015). Ion-ion plasma consists of positive and negative ions with a negligible number of electrons. Such plasma is generally observed when the electronegativity is very high and the dynamics of the plasma determined only by the heavy stationary ions (Oudini et al., 2013).

Plasma with both ion species having comparable mass and temperature show different behavior than the conventional electron-ion plasma. This difference in properties has wide implications in the fabrication of semiconductor devices in the electronics industry (Kanakasabapathy et al., 2001) and the design of efficient plasma propulsion with thruster that provides thrust by accelerating alternatively positive and negative charged ions (Aanesland et al., 2009). Recently, it has been validated experimentally that both positive and negative ion beams can be successfully accelerated to provide thrust in PEGASES, in which $\mathrm{SF}_{6}$ was used as working electronegative gas (Rafalskyi et al., 2014). Midha et al. (2002) studied the response of ion-ion plasma on applied DC bias with respect to time.
The study was based on the time-dependent onedimensional fluid model and it was sufficed that unlike conventional plasma, the potential of the plasma does not lie towards one of the electrodes. Meige et al. (2008) incorporated the PIC simulation method to investigate the properties of the ion-ion plasma. The obtained results were congenial with previous work by Midha et al. (2002). Besides, the existence of a presheath region was observed upon keen inspection of the potential profile at the bulk plasma. The ion-ion plasma potential plays a key role in the extraction and acceleration of ions in PEGASES thruster (Popelier et al., 2011).

One of the interesting characteristics of ion-ion plasma is the presence of minute, almost zero electrostatic fields in the bulk plasma region unlike the conventional plasma (Walton et al., 2003). However, Meige et al. (2008) reported that there exists a small magnitude of finite electrostatic potential $(0.01 \mathrm{~V})$ similar to the conventional plasma, which accelerates the ion before entering the sheath region; suggests equivalence of Bohm criterion in ion-ion plasma. Generally, it was observed that in the absence of bias, the ions flow towards the electrode at an equal rate (Economou, 2007). Diop et al. (2019) studied the increase in argon ionization, in low power thruster, with the addition of a significant amount of xenon gas in the argon flow. It was found that the ion's kinetic energy and current generated by the thruster increased which is useful in designing the space propulsion and ion source applications. Thakur et al. (2018) studied the conventional electron-ion plasma in cylindrical plasma 
system using fluid approach in which the effect of oblique magnetic field on plasma sheath characteristics in both collisional and collisionless cases were presented. Basnet and Khanal (2020) studied the electronegative magnetized plasma-wall transition characteristics using a kinetic trajectory simulation method. It was found that the temperature distribution of negatively charged particles was non-uniform in the sheath region which determined the energy flux flow towards the wall.

In this work, the effect of DC bias and ion temperature on bounded ion-ion plasma was investigated by using a fully self-consistent one-dimensional electrostatic PIC simulation method. The motion of charged particles is influenced by the bias voltage and ion temperature which implicitly affect the plasma sheath and presheath structures. The strength of the electrostatic electric field increases with the increase in biasing voltages; however, nearly zero-field exits at the center of the ion-ion plasma. The quasineutral property of ion-ion plasma vanishes after $1000 \mathrm{~V}$. The temperature of ion has an inverse effect on the electric field as the height of the electric field decreases for the increase in ion temperature. The effect of temperature and biasing voltage on the particle densities and ion distribution are systematically studied which is useful in electrostatic ion thruster.

\section{MATERIALS AND METHODS}

In the PIC method, the positions and velocities of the particles are described in continuous phase space and the plasma quantities like densities, fields, potentials are assigned to stationary mesh points (Timko, 2011). In a real plasma system, the number of plasma particles is very large, and keeping track of these particles in the simulation is a computationally expensive and tedious job. Hence, PIC adopts the concept of super particles, which comprises of many real particles at the same time. The concept of super particles was adopted for the particle simulation because the Lorentz force, which is essential in describing the dynamics of plasma species, depends only on the charge to mass ratio, and is the same for both real and super particles (Birdsall \& Langdon, 2004). Each particle was tracked throughout the simulation; hence, PIC gives the complete description of plasma dynamics. In the present work, the algorithm mainly consists of two parts: initiation and iteration. In initiation part, the ions were volumetrically loaded into the simulation region with a random distribution of initial positions while velocities were assigned from a Maxwellian distribution defined by equation (1) truncated at below $\sigma= \pm 4$ with $\sigma=1 / \sqrt{2} v_{\text {th }}$, where $v_{\text {th }}$ is the thermal velocity of ion species (Cartwright et al., 2000). The onedimensional particle distribution function takes the form

$$
f(v) \propto \exp \left(-\frac{v^{2}}{v_{t h}^{2}}\right)
$$

After the initiation was complete, the charge and current densities were weighted to the grid points from the particle positions and self-consistent potentials were obtained at the grid points by solving the Poisson's equation. Electric fields were then calculated and subsequently, a force on each particle was obtained using a similar weighting scheme as used for densities. This was done to avoid the self-force i.e., a particle accelerating itself (Tskhakaya et al., 2007). After this, the particles were moved forward in time-solving equations of motion. This procedure was repeated through the same cycle up to a specified number of time steps. Due to the presence of boundary walls, appropriate particle boundary conditions were included in the simulation and the absorption and reflection boundary conditions were incorporated for the ions. The reflection velocity of ions from the boundary was used as defined by equation (2). An injection scheme was used in which ions were injected with random positions assigned from a uniform random distribution with appropriate velocities. Time centering of these positions and velocities was done to maintain the secondorder accuracy akin to leap-frog integrator used in this work. Similarly, when the ions were reflected from the boundaries, a simple specular reflection was used where it was envisaged that a particle's intrinsic properties remain unaltered when it interacts with the boundary with only change of its direction. The velocity of the reflected particle is given as (Tskhakaya et al., 2007);

$$
v_{r e f}=-v+\left|\frac{x}{v}\right| \frac{Q}{m} E_{x}
$$

where $v_{\text {ref }}$ is the velocity of the reflected ion into the simulation region, $v$ is the velocity of ion and $E_{x}$ is electric field experienced by ion in the simulation region, $Q$ is the charge of the superparticle and $m$ is its mass.

The equation describing the motion of particles is

$$
m \frac{d \vec{v}}{d t}=Q \vec{E}
$$

The Poisson's equation that connects the electrostatic electric field $\vec{E}$ and charge density $\rho$ is

$$
\nabla \cdot \vec{E}=\frac{\rho}{\varepsilon_{0}}
$$

where $\varepsilon_{0}$ is the permittivity of free space. To obtain the potential distribution from the Maxwell equation (4), we use the finite-difference method.

The ion equation of motion, as given in equation (3) above is solved for the particles using the particle mover scheme in which the phase space is discretized as (Tskhakaya et al., 2007) 


$$
\frac{x_{k+1}-x_{k}}{d t}=v_{k+1 / 2}
$$

and

$$
\frac{v_{k+1 / 2}-v_{k-1 / 2}}{d t}=\frac{F_{k}}{m}
$$

where the subscript ' $k$ ' in equations (5) and (6) refers to old quantities from the previous time step and ' $k+1$ ' refers to newly updated quantities for the next time step.

The ion-ion plasma system was bounded by two equal and opposite biased walls as shown in Fig. 1. The positive ions were reflected from the positively biased wall and were absorbed in the negatively biased wall. On the other hand, negative ions were reflected from the negatively biased wall and absorbed at the positively biased wall.

\section{Plasma}

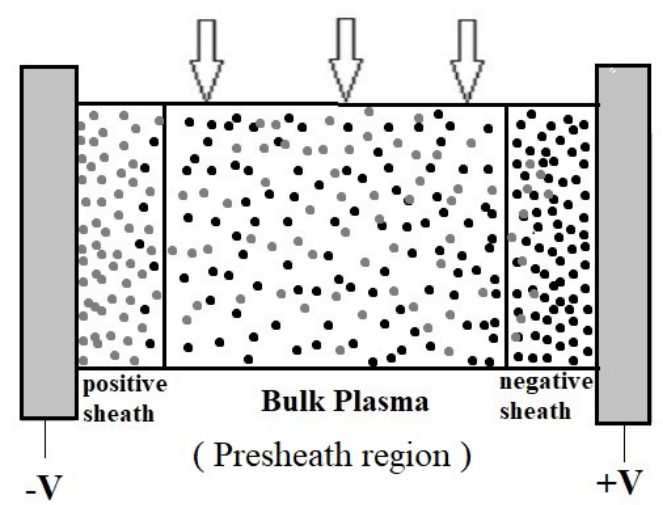

Fig. 1. A schematic diagram of the one-dimensional DC biased ion-ion plasma system

To maintain the stability of the simulation, we adopt the stability conditions as $\Delta x \leq \lambda_{D n}$ and $d t \omega_{p}=0.1$, in which $\Delta x$ is the size of the simulation cell, $\lambda_{D n}$ is the Debye length as defined by equation (8), $d t$ is the numerical time step in the simulation and $\omega_{p}$ is the plasma frequency. Due to the negligible number of electrons, the ion-ion plasma characteristics solely depend on the dynamics of heavy ions. The Debye length is an important parameter, which measures the shielding thickness of the plasma sheath. In the case of electronegative plasma with electrons, the Debye length of the system is given as

$$
\frac{1}{\lambda_{D}}=\sqrt{\left(\frac{\delta_{e}}{T^{e}}+\frac{\delta_{n}}{T^{n}}+\frac{1}{T^{p}}\right) \frac{n_{p} e^{2}}{\varepsilon_{0} k_{B}}}
$$

in which $\delta_{e}=n_{e} / n_{p}$ is the ratio of the concentration of electron $\left(n_{e}\right)$ to positive ion $\left(n_{p}\right), \delta_{n}=n_{n} / n_{p}$ is the ratio of the concentration of negative ions $\left(n_{n}\right)$ to positive ions $\left(n_{p}\right), T^{e}, T^{n}$ and $T^{p}$ are the temperatures of the electron, negative and positive ions, respectively.

For the ion-ion plasma $\delta_{e}=0$ and $\delta_{n}=1$, so the Debye length of the system equation (7) becomes

$$
\frac{1}{\lambda_{D n}}=\sqrt{\left(\frac{1}{T^{n}}+\frac{1}{T^{i}}\right) \frac{n_{p} e^{2}}{\varepsilon_{0} k_{B}}}
$$

Equation (8) shows that the Debye length depends on the temperature of negative and positive ions.

\section{RESULTS AND DISCUSSION}

The numerical results obtained by solving the related kinetic equations via electrostatic PIC simulations of the ion-ion plasma with biasing boundaries have been presented. The plasma consisted of only positive and negative ions having a mass $40 \mathrm{amu}$, two different temperatures ( 1 and 3 ) eV, and a plasma density of $10^{15}$ $\mathrm{m}^{-3}$. The number of super particles was $10^{4}$ and the length of the simulation region was $2.18 \mathrm{~cm}$. The simulation region was discretized into 100 cells. The obtained plasma properties are time-averaged for $15 \mu$ s (2000 time steps). The plasma discharge with argon gas (40 amu) is widely used in various plasma applications, for example, to clean the surface of materials, physical sputtering, and implantation as argon is chemically inert and relatively cheap. Moreover, argon discharge is useful in plasma thruster in the significant presence of xenon gas (Diop et al., 2019).

The phase space diagram of positive and negative ions is shown in Figs. 2 and 3, respectively. In Figs. 2 and 3, the negative and positive velocities represent the motion of positive and negative ions directed towards the negatively and positively biased electrodes, respectively. The distance was normalized in terms of Debye length defined by equation (8). When the biasing voltage was $100 \mathrm{~V}$, it was seen that most of the ions were ensnared in the bulk plasma and only those ions above critical velocity can reach the respective electrodes i.e., positive ions can reach the negatively biased electrode (Fig. 2a) and negative ions can reach the positively biased electrodes (Fig. 3a). But when the biasing voltage increased by 10 times, most of the positive ions leave the quasineutral region and flow towards the left-hand electrode (Fig. 2b) whereas the negative ions leave the quasineutral region and flow towards the right-hand electrode (Fig. 3b). However, a certain fraction of positive and negative ions were present at the positively and negatively biased electrodes respectively. Most of these positive ions have negative velocities and negative ions have positive velocities and the magnitude of these velocities get increased with the increase in biasing voltage. This suggests that the ions after reaching the biasing wall get reflected in the simulation region. The 
phase space plot of positive and negative ions clearly shows that sheaths are formed due to the extraction of ions by the biasing voltages. Due to the residual electric field at the bulk plasma, both positive and negative ions were extracted in approximately equal amounts by the biasing voltages.

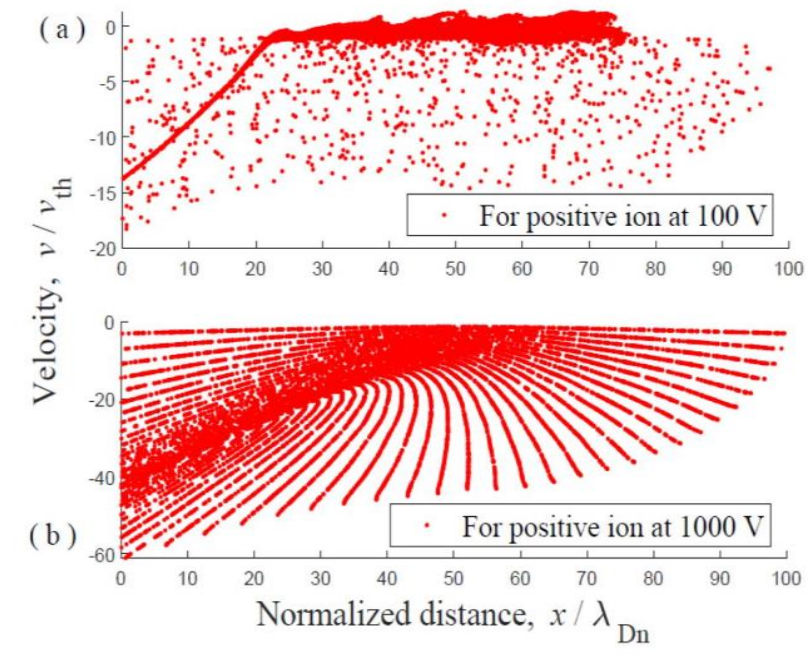

Fig. 2. Phase space diagram of positive ions for two different biasing voltages (a) $100 \mathrm{~V}$ and (b) $1000 \mathrm{~V}$

To understand the sheath and presheath formation in bounded ion-ion plasma, we present the electric field profile for different values of biasing voltage and ion temperature as shown in Fig. 4. The height of the electric field barrier gets increased (Fig. 4a) with the increase of bias voltages, which enforces the trapping of more charged particles in bulk plasma. The magnitude of the electric field of quasineutral plasma was almost halfway between the two biased electrodes unlike electron-ion plasma, which was almost constant at the center suggesting nearly zero electric fields, except for the case of $1000 \mathrm{~V}$ biasing voltage. In this exceptional case, a strong electric field penetrates deep into the bulk plasma so that the plasma does not remain quasineutral to a large extent, as usual; and also can no more behave collectively. Upon close inspection, it can be observed that there exists a finite electric field in the quasineutral region, which is depicted in the blow-up Fig. 4(b). Therefore, there exists a presheath region that accelerates the ion to a critical velocity with which it enters into the sheath region as similar to conventional electron-ion plasma. The result is qualitatively in agreement with the previous work of Meige et al. (2008). When the temperature of ion-ion plasma increases, the bulk plasma starts to dominate the sheath thickness, which is illustrated in Fig. 4(b). The control electric field on ionion plasma is very useful for the alternately extraction and acceleration of positive and negative ions to provide the thrust in the PEGASES thrusters.

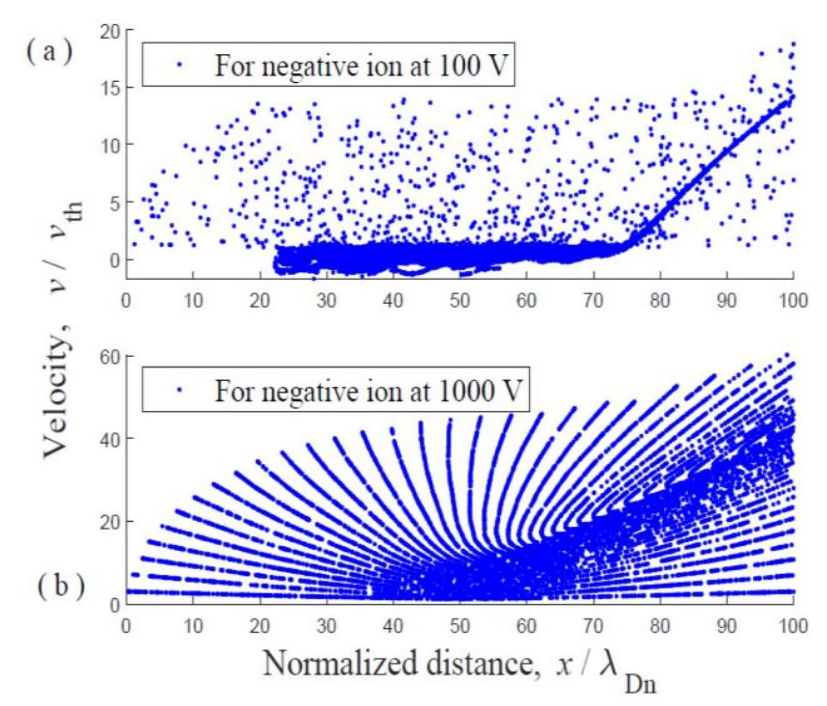

Fig. 3. Phase space diagram of negative ions for two different biasing voltages (a) $100 \mathrm{~V}$ and (b) 1000
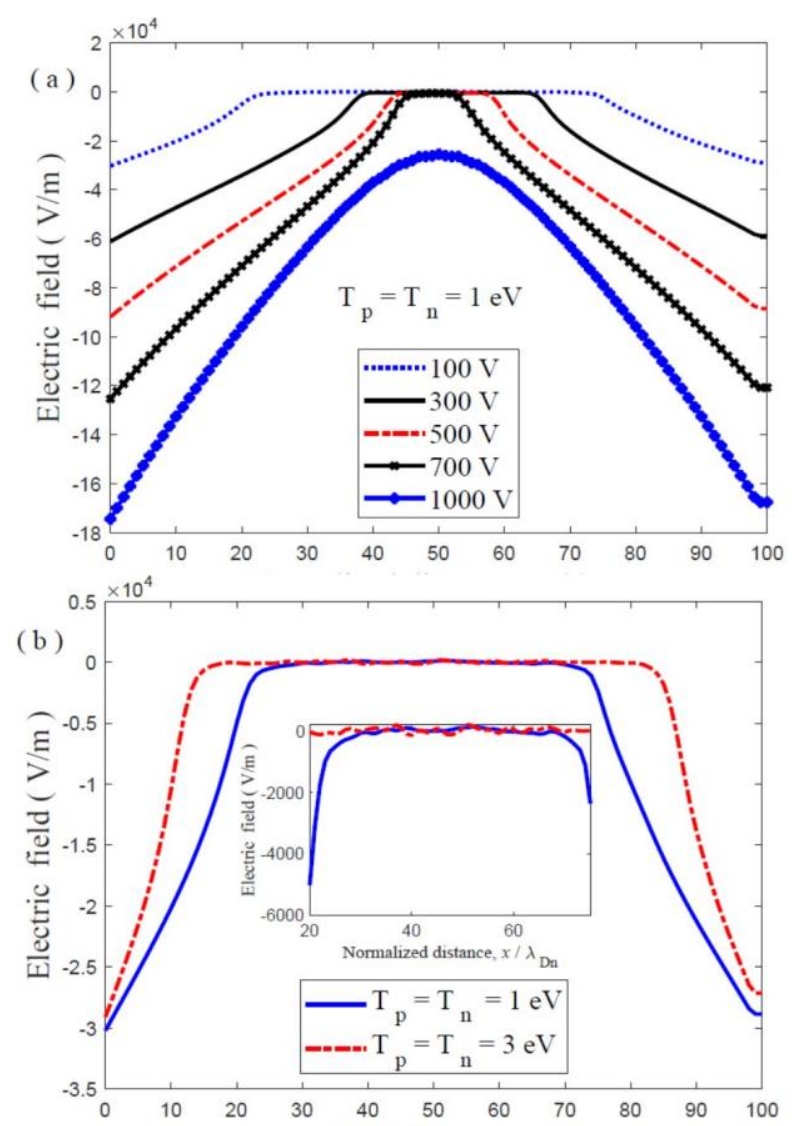

Fig. 4. Electrostatic electric field profile (a) for different biasing voltages (b) for two different temperatures at constant biasing voltage $100 \mathrm{~V}$

The space charge density is affected by the electrode biasing voltage and temperature of ions. The variation of space charge density as the function of normalized distance is shown in Fig. 5. As the plasma was 
quasineutral at the core region, the space charge density of the ion-ion plasma was almost zero. The width of this region decreased with the increase in biasing voltage and hence, the quasineutral region vanished after $1000 \mathrm{~V}$, which is illustrated in Fig. 4(a). The space charge density towards the left-hand electrode was always positive to shield the effect of applied negative biasing potential while it was always negative towards the right-hand electrode to shield the positive biasing potential. The magnitude of space charge density decreases with the increase in temperature of ions as shown in Fig. 5(b).
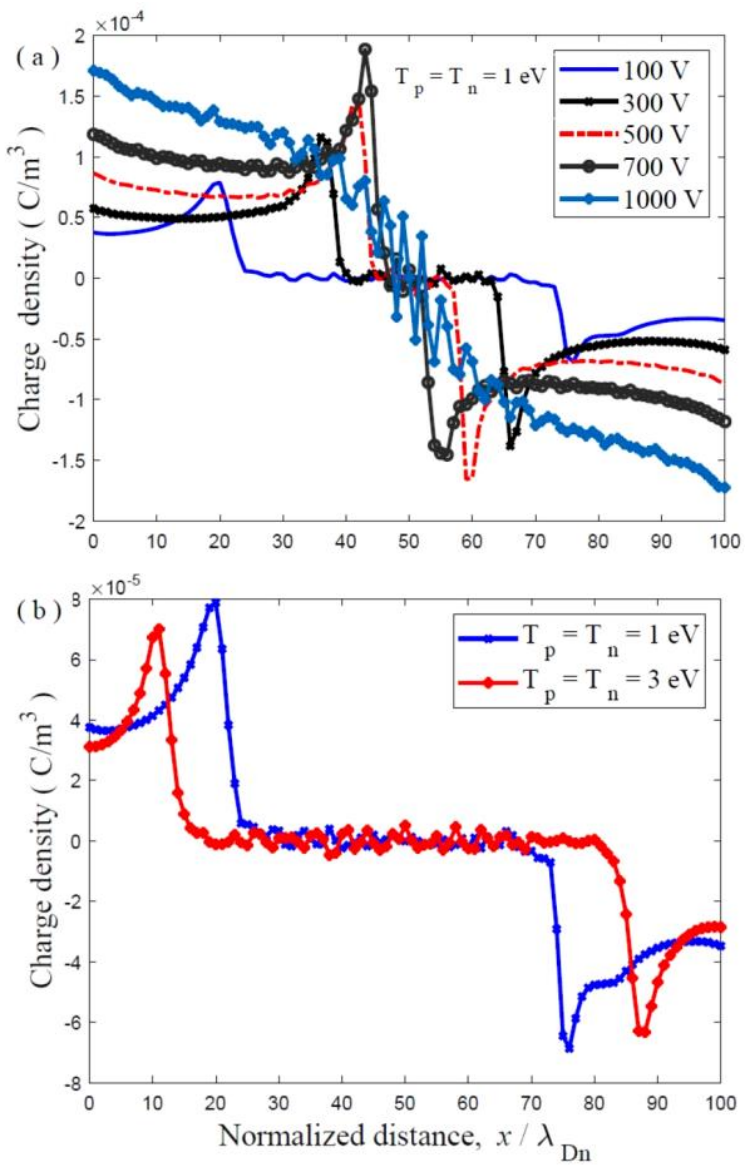

Fig. 5. Space charge density profile (a) for the different biasing voltages (b) for two different temperatures at constant biasing voltage $100 \mathrm{~V}$

The variation of ion densities as the function of normalized distance is depicted in Fig. 6. It was found that the number density of the positive ions was much more than that of negative ions at the left electrode and vice-versa at the right electrode. The density of ions was higher in the presheath region; however, it goes on decreasing towards the sheath region. Also, it was observed that the density of ions at the bulk plasma increased with the increase in electrode biasing voltage (Fig. 6a) and it decreased with the increase in ion temperature of ion-ion plasma (Fig. 6b). The increment of the height of the potential barrier with the increase in biasing voltage traps more ions in the bulk region. The positive and negative ion density profiles exhibit similar nature at the quasineutral region (core plasma), which was due to their same mass and temperature.
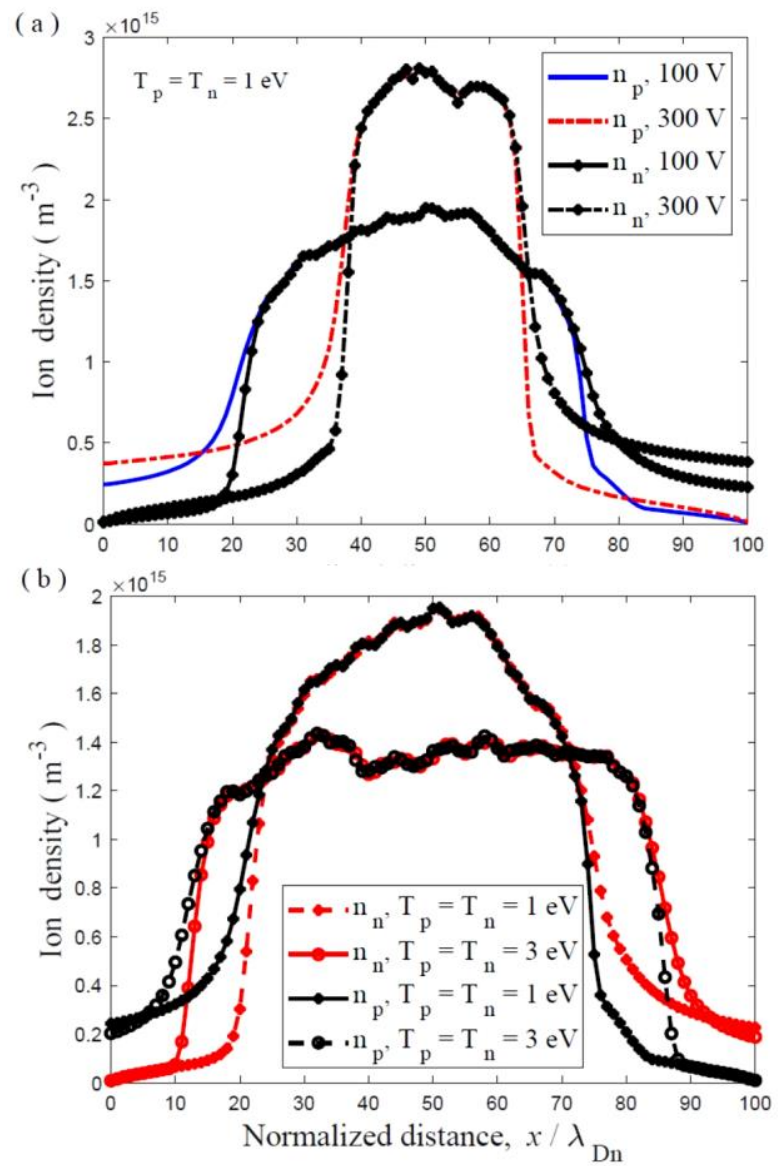

Fig. 6. Ion densities profile (a) for two different biasing voltages (b) for two different temperatures at constant biasing voltage $100 \mathrm{~V}$

The final time step velocity distributions of positive and negative ions within a velocity range $d v$ for the different electrode biasing voltages are depicted in Figs. 7 and 8, respectively. It is seen that most of the negative ions have a positive velocity towards the right-hand electrode while the positive ions have a negative velocity towards the left-hand electrode. However, a minute fraction of positive ions have positive velocities (Fig. 7) and negative ions have negative velocities (Fig. 8). From the phase space plot shown in Figs. 2 and 3, these ions were found in the quasineutral region. This shows that there is fluctuation in the quasineutral region, due to the effect of sheaths formed near the electrodes. The positive sheath attracts negative ions of the bulk near to its vicinity. However, these ions cannot reach the left-hand electrode and were reflected in the simulation region. A similar phenomenon was observed for the negative ions in the 
vicinity of the positive sheath. Therefore, ions were trapped in the bulk plasma region. More ions were accelerated from the bulk region towards the sheath which gets increased with the increase in biasing voltage. Therefore, the number of superparticles having maximum velocity increased with the increase in the biasing voltage.

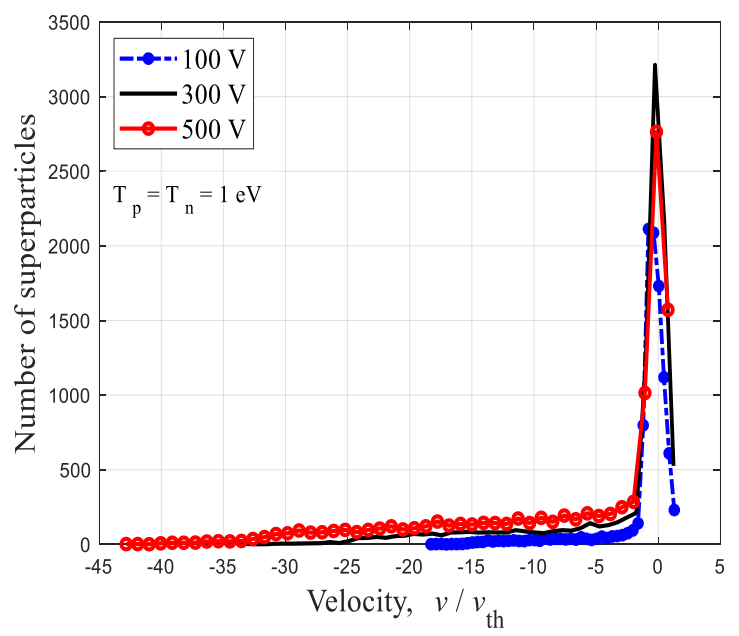

Fig. 7. Positive super ion velocity distribution for the different biasing voltages

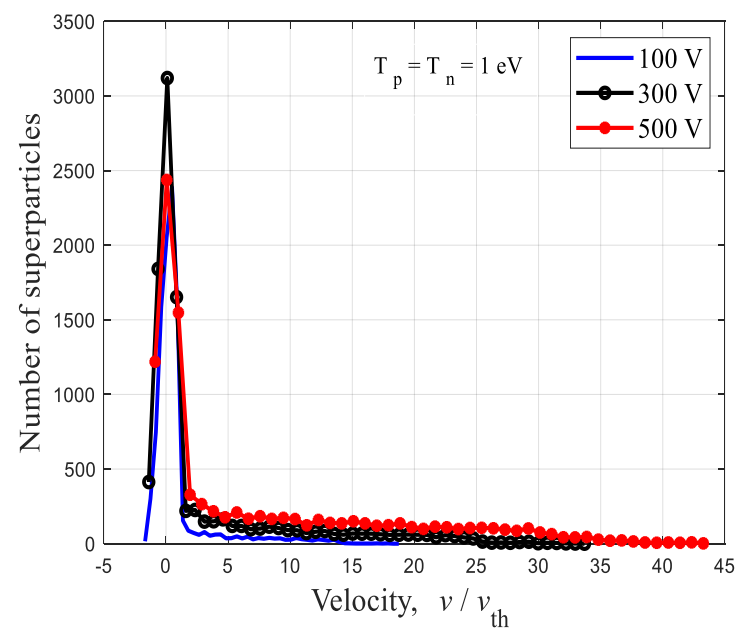

Fig. 8. Negative super ion velocity distribution for the different biasing voltages

\section{CONCLUSIONS}

The dynamics of bounded ion-ion plasma was investigated by using the PIC simulation method for different values of biasing voltage and ion temperature. The ions were trapped in the bulk plasma and only those above critical velocity can reach to the oppositely biased electrodes, i.e., the negative ions reach the positive electrode while positive ions reach the negative electrode. This mechanism ensures the formation of plasma sheaths at both electrodes. The electric field profile was constant in the middle having nearly zero fields which decreased monotonically towards the electrodes on either side. As the biasing voltage increases, the height of the electric field barrier increases, and more charged particles are trapped in the bulk plasma whereas the increment of temperature has an inverse effect on the barrier height. Therefore, the fluctuation of the electric field with biasing voltage and temperature affects the core plasma and space charge density as well. The space charge density was zero in the bulk plasma but its magnitude increased towards the electrode. The quasineutral property of plasma vanished at electrode biasing voltage of $1000 \mathrm{~V}$. The density of positive ions was higher than the negative ions at the negatively biased electrode and vice-versa at the positively biased electrode. Besides, it was observed that the density of ions at the bulk plasma increased with the increase in the biasing voltage and decreased with the increase in ion temperature. The positive ions acquired negative velocity towards the negatively biased electrode and the negative ions acquired positive velocity towards the positively biased electrode. These properties of ionion plasma can be exploited to control the flow of particles towards the boundary by varying the biasing voltage and ion temperatures, leading to useful applications in electrostatic electric propulsion thruster, dry etching process and to clean the surface by sputtering.

\section{ACKNOWLEDGMENTS}

S. Basnet would like to acknowledge the University Grants Commission, Sanothimi, Bhaktapur, Nepal for the $\mathrm{Ph} . \mathrm{D}$. Fellowship through the grant number $\mathrm{PhD} / 075-76-$ S\&T-16.

\section{REFERENCES}

Aanesland, A., Meige, A., \& Chabert, P. (2009). Electric propulsion using ion-ion plasmas. Journal of Physics: Conference Series, 162(1), 012009-13pp.

Basnet, S., \& Khanal, R. (2020). Kinetic simulation of an electronegative plasma with a cut-off distribution and modified Bohm criterion. Plasma Science and Technology, 24(4), 045001-7pp.

Birdsall, C. K., \& Langdon, A. B. (2004). Plasma physics via computer simulation. New York, London: Taylor \& Francis Group, LLC.

Cartwright, K. L., Verboncoeur, J. P., \& Birdsall, C. K. (2000). Loading and injection of Maxwellian distributions in particle simulations. Journal of Computational Physics, 162(2), 483-513.

Chapagain, N. P. (2015). Dynamics ionospheric plasma bubbles measured by optical imaging system. Journal of Institute of Science and Technology, 20(1), 20-27. 
Diop, F., Gibert, T., \& Bouchoule, A. (2019). Argon ionization improvement in a plasma thruster induced by a few percents of xenon. Physics of Plasmas, 26(6), 063508-11pp.

Economou, D. J. (2007). Fundamentals and applications of ion-ion plasmas. Applied Surface Science, 253(16), 6672- 6680 .

Economou, D. J. (2014). Pulsed plasma etching for semiconductor manufacturing. Journal of Physics D: Applied Physics, 47(30), 30300-27pp.

Franklin, R. N. (2002). Electronegative plasma-why are they so different? Plasma Sources Science Technology, 11(3A), A31-A37.

Kanakasabapathy, S. K., Overzet, L. J., Midha, V., \& Economou, D. (2001). Alternating fluxes of positive and negative ions from an ion-ion plasma. Applied Physics Letters, 78(1), 22-24.

Meige, A. Leray, G., Raimbault, J. L., \& Chabert, P. (2008). Sheath and presheath in ion-ion plasmas via particle-in-cell simulation. Applied Physics Letters, 92(6), 061501-3pp.

Midha, V., Ramamurthi B., \& Economou, D. J. (2002). Time evolution of an ion-ion plasma after the application of a direct current bias voltage. Journal of Applied Physics, 91(10), 6282-6287.

Oudini, N., Raimbault, J. L., Chabert, P., Meige, A., \& Aanesland, A. (2013). Particle-in-cell simulation of an electronegative plasma under direct current bias studied in a large range of electronegativity. Physics of Plasmas, 20(4), 043501-7pp.

Popelier, L., Aanesland, A., \& Chabert, P. (2011). Response of ion-ion plasma to DC biased electrodes. Journal of Physics D: Applied Physics, 44(31), 053301-10pp.

Rafalskyi, D., Popelier, L., \& Aanesland, A. (2014). Experimental validation of the dual positive and negative ion beam acceleration in the plasma propulsion with electronegative gases thruster. Journal of Applied Physics, 115(5), 053301-9pp.

Thakur, P. K., Pokhrel, R. R., \& Khanal, R. (2018). Fluid analysis of magnetized plasma sheath in a cylindrical geometry. Journal of Institute of Science and Technology, 23(1), 26-29.

Timko, H. (2011). 2D Arc-PIC code description: methods and documentation. CERN-European Organization for Nuclear Research, Geneva, Switzerland, CLIC Notes 1032.

Tskhakaya, D., Matyash, K., Schneider, R., \& Taccogna, F. (2007). The particle-in-cell method. Contribution to Plasma Physics, 47(8-9), 563-594.

Walton, S. G., Leonhardt, D., Fernsler, R. F., \& Meger, R. A. (2003). On the extraction of positive and negative ions from electron-beam-generated plasmas. Applied Physics Letters, 83(4), 626-628. 